\title{
Tuning Anti-Klein to Klein Tunneling in Bilayer Graphene
}

\author{
Renjun Du (杜人君) ${ }^{1, *}$ Ming-Hao Liu (劉明豪),${ }^{2,3, \dagger}$ Jens Mohrmann, ${ }^{1}$ Fan Wu (吴凡), ${ }^{1,4}$ \\ Ralph Krupke, ${ }^{1,5}$ Hilbert von Löhneysen, ${ }^{1,6}$ Klaus Richter, ${ }^{3}$ and Romain Danneau ${ }^{1, \$}$ \\ ${ }^{1}$ Institute of Nanotechnology, Karlsruhe Institute of Technology (KIT), D-76021 Karlsruhe, Germany \\ ${ }^{2}$ Department of Physics, National Cheng Kung University, Tainan 70101, Taiwan \\ ${ }^{3}$ Institut für Theoretische Physik, Universität Regensburg, D-93040 Regensburg, Germany \\ ${ }^{4}$ College of Optoelectronic Science and Engineering, National University of Defense Technology, Changsha 410073, China \\ ${ }^{5}$ Institute of Material Science, Technische Universität Darmstadt, D-64287 Darmstadt, Germany \\ ${ }^{6}$ Institute for Solid State Physics and Physics Institute, Karlsruhe Institute of Technology (KIT), D-76021 Karlsruhe, Germany
}

(Received 22 March 2017; revised manuscript received 15 July 2018; published 21 September 2018)

\begin{abstract}
We show that in gapped bilayer graphene, quasiparticle tunneling and the corresponding Berry phase can be controlled such that they exhibit features of single-layer graphene such as Klein tunneling. The Berry phase is detected by a high-quality Fabry-Pérot interferometer based on bilayer graphene. By raising the Fermi energy of the charge carriers, we find that the Berry phase can be continuously tuned from $2 \pi$ down to $0.68 \pi$ in gapped bilayer graphene, in contrast to the constant Berry phase of $2 \pi$ in pristine bilayer graphene. Particularly, we observe a Berry phase of $\pi$, the standard value for single-layer graphene. As the Berry phase decreases, the corresponding transmission probability of charge carriers at normal incidence clearly demonstrates a transition from anti-Klein tunneling to nearly perfect Klein tunneling.
\end{abstract}

DOI: 10.1103/PhysRevLett.121.127706

Introduction.-Bilayer graphene (BLG), like its singlelayer counterpart [1-4], exhibits outstanding physical properties [5-8] and is often regarded as a promising material for potential electronic applications. One striking feature of BLG is the possibility to induce and tune an electronic band gap by breaking the lattice inversion symmetry using, for example, an electric field [7-11]. However, our fundamental knowledge of the gapped states in BLG remains limited in many respects despite the existing studies of the Berry phase [6,12-15] and quasiparticle tunneling [14,16-18].

The emergence of a band gap has a strong impact on the Berry phase by modulating the pseudospin $\sigma[19,20]$, which expresses an extra quantum mechanical degree of freedom in graphene [1,16]. In Figs. 1(a) and 1(b), the pseudospin vectors at different Fermi levels are depicted as small cones and projected in a plane between the conduction (yellow) and valence (blue) bands in the momentum space. After a pseudospin vector adiabatically travels a closed path around the valley, e.g., the red circle in Figs. 1(a) and 1(b), a Berry phase is acquired [15,21-23]. This process is better visualized on a Bloch sphere, as shown in Figs. 1(c) and 1(d), where the pseudospin (denoted by arrows) traces out a solid angle which is equivalent to the Berry phase of BLG [15,21]. In the absence of a band gap, e.g., in pristine BLG, the pseudospin vector always lies in the plane [20] [see Figs. 1(a) and 1(c)], so the corresponding Berry phase remains $2 \pi[6,8]$, as shown by the half-spherical surface in Fig. 1(c). On the other hand, the pseudospin may be polarized out of plane

$[15,19,20,22,24]$ in gapped BLG [see Figs. $1(\mathrm{~b})$ and $1(\mathrm{~d})]$, leading to a Berry phase in the range of $0-2 \pi$ as shown in Fig. 1(d). The understanding of the tunable Berry phase in gapped BLG may shed light on the physical phenomena, such as the valley Hall effect [25-28], the anomalous Hall effect $[29,30]$, and quasiparticle tunneling [14,15].
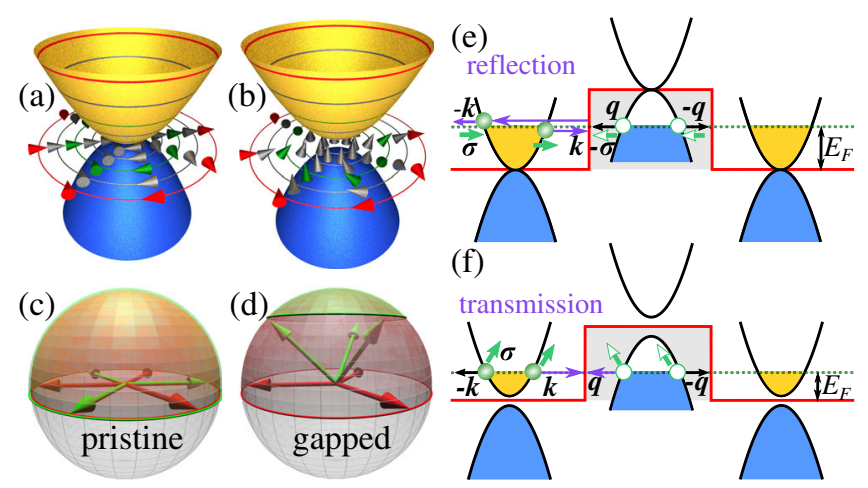

FIG. 1. Sketches of band structure and pseudospin orientation for (a) pristine and (b) gapped BLG. Pseudospin vectors at different Fermi levels (contours) are projected as small cones on a plane between the conduction (yellow) and valence (blue) bands. (c) and (d) show the corresponding Berry phase as the solid angle traced out by the pseudospin (arrows) on the Bloch sphere for (a) and (b), respectively. The red (green) color in (a)-(d) refers to high (low) Fermi energy $\left(E_{F}\right)$. (e) Anti-Klein tunneling for pristine BLG. $k$ or $q$ is the wave vector for electrons or holes, and $\boldsymbol{\sigma}$ denotes the pseudospin. (f) Klein tunneling is possible in gapped BLG. 
A comprehensive exploration of the Berry phase in gapped BLG is, therefore, of fundamental interest.

The band gap also significantly affects quasiparticle tunneling, which is associated with the pseudospin [16] and the Berry phase $[14,15]$. The quasiparticle tunneling in pristine BLG exhibits perfect reflection when the charge carriers encounter a sharp potential barrier at normal incidence, an effect known as anti-Klein tunneling [16,18], as illustrated in Fig. 1(e). However, when the band gap opens, anti-Klein tunneling can be reduced while the Berry phase slightly changes [14]. Indeed, perfect Klein tunneling, i.e., full transmission through a potential barrier $[16,17,31-33]$, may be possible in gapped BLG due to the out-of-plane polarization of the pseudospin [15] [see Fig. 1(f)]. However, the observation of Klein tunneling in gapped BLG requires low-disorder devices and ballistic transport. To the best of our knowledge, such an anti-Klein to Klein tunneling transition has not been observed in BLG.

In this Letter, we employ an edge-connected hBN-BLG$\mathrm{hBN}$ heterostructure (where $\mathrm{hBN}$ is hexagonal boron nitride) to investigate quasiparticle tunneling in a lateral pnp junction. We benefit from an advanced sample fabrication method [34], yielding ultraclean devices, which enable ballistic Fabry-Pérot (FP) interferences [35]. The phase-sensitive FP interference is used to detect the variation of the Berry phase. In contrast to previous work examining the Berry phase merely at high Fermi energies [14], the robust FP interference allows us to probe it close to the band edge. The role of the Berry phase and of the corresponding pseudospin on the quasiparticle tunneling will be discussed in detail and compared to numerical simulations based on a tight-binding model [36].

Sample description.-The investigated devices, sketched in Fig. 2(a), consist of a hBN-BLG-hBN heterostructure. The encapsulation of BLG results in low-disorder devices, allowing ballistic transport over a distance of $9 \mu \mathrm{m}$. The potential profile across the device is controlled by a local top gate about $150 \mathrm{~nm}$ wide, as well as a global back gate (Si substrate). The fabrication follows Ref. [34]. Details of the devices are shown in the Supplemental Material [37]. Each device is divided into four regions, labeled as $T$ (topand back-gated region), $B$ (only back-gated regions), and $C$ (contact-overlapping region) in Fig. 2(b). The overlapping contact results in additional $n$-doping in region $C$ when both gates are set to zero, as displayed in Fig. 2(e), where the carrier density profile is obtained from finite-elementbased electrostatic simulation using FEnics [47] combined with the mesh generator Gmsh [48].

Fabry-Pérot interferences.-The conductance $(G)$ as a function of the top-gate $\left(V_{\mathrm{tg}}\right)$ and back-gate $\left(V_{\mathrm{bg}}\right)$ voltages has been probed experimentally and modeled for device PNJ-A, as shown in Figs. 2(c) and 2(d), respectively. The conductance minima appear as three lines in these plots. The two horizontal lines at $V_{\mathrm{bg}} \approx-1.3 \mathrm{~V}$ and $V_{\mathrm{bg}} \approx-26.9 \mathrm{~V}$ are independent of $V_{\mathrm{tg}}$ and indicate the charge neutrality
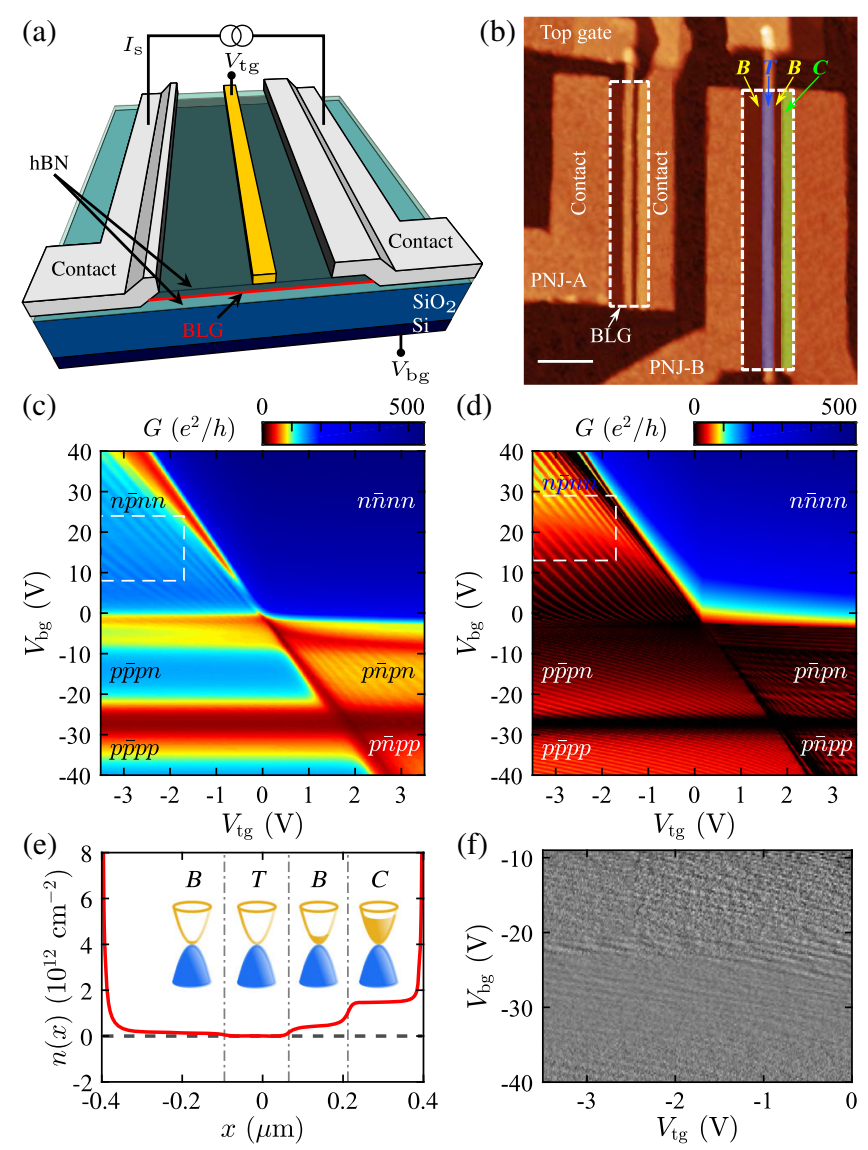

FIG. 2. (a) Sketch and (b) AFM image of the devices. Scale bar in (b) is $1 \mu \mathrm{m}$. (c) Experimental and (d) simulation results of conductance $G$ varying with $V_{\mathrm{tg}}$ and $V_{\mathrm{bg}}$ at $4.2 \mathrm{~K}$ and zero magnetic field for device PNJ-A. (e) The initial charge-carrier density $n(x)$ across device PNJ-A when $V_{\mathrm{bg}}$ and $V_{\mathrm{tg}}$ are both zero. (f) Transconductance $d G / d V_{\mathrm{tg}}$ in the $p \bar{p} p n$ and $p \bar{p} p p$ regions of (c).

point (CNP) in regions $B$ and $C$, respectively. The position of the CNP is determined by the initial doping of each region [see Fig. 2(e)]. The diagonal line shows the CNP of the dual-gated region $T$ and defines the displacement field axis, along which the interlayer asymmetry develops. The three lines partition the map into six sections, each of which has a unique combination of charge carrier polarities, as labeled in Figs. 2(c) and 2(d).

FP interferences arise in an electrostatic potential barrier with two semitransmitting interfaces if the phase difference $\Delta \Phi$ between two neighboring transmitted waves fits the resonance condition $\Delta \Phi=2 \pi j$ (where $j$ is integer). In the bipolar regime ( $n \bar{p} n n, p \bar{n} p n$, and $p \bar{n} p p)$, where the charge-carrier type in region $T$ (denoted by the overlined symbol) is different from the adjacent region $B$, we observe clear conductance oscillations as a consequence of FP interferences. The FP fringes extend along the diagonal line, illustrating that the FP interference occurs in a cavity tuned by both $V_{\mathrm{tg}}$ and $V_{\mathrm{bg}}$. The cavity length is determined by the resonance condition of FP interferences as in 
Ref. [14], and is found to be around $150 \mathrm{~nm}$, which corresponds to the top-gate width. On the other hand, due to the long spacing between the contacts, FP interferences in unipolar regimes such as $p \bar{p} p n$ and $p \bar{p} p p$ are hardly visible. However, the weak oscillations become discernible in the transconductance $d G / d V_{\operatorname{tg}}$ gate map; see Fig. 2(f). More details about FP interferences are shown in the Supplemental Material [37].

To gain further insight into the implications and ramifications of our experimental results, quantum transport simulations based on the real-space Green's function method using the tight-binding model for Bernal-stacked BLG have been performed. Details of the simulation method are similar to those in Ref. [14], including how the gate-tunable interlayer asymmetry parameter $U$ can be implemented [8], with the following two alterations: First, the scalable tight-binding model [49] with a scaling factor of $s_{f}=4$ has been adopted. Second, carrier density profiles obtained from electrostatic simulations [an example is shown in Fig. 2(e)] have been implemented in order to extract realistic on-site energy profiles for the tight-binding model Hamiltonian. More details about the gate-modulated carrier density profiles can be found in the Supplemental Material [37]. Comparing Figs. 2(c) and 2(d), our experiment captures all the interference patterns that are theoretically predicted. This agreement demonstrates the high quality of both our FP interferometer design and the quantum transport simulations, even comparable to the suspended graphene interferometer with smooth junction profiles that led to high FP finesse [35].

Berry phase and quasiparticle tunneling.-At low magnetic fields, the phase difference $\Delta \Phi$ comprises not only the conventional kinetic part, the Wentzel-Kramers-Brillouin phase $\Phi_{\mathrm{WKB}}$, but also the Aharonov-Bohm phase $\Phi_{\mathrm{AB}}$ and the Berry phase $\Phi_{\text {Berry }}$, which may arise under magnetic fields. The effect of the Berry phase on FP interferences may manifest itself as phase shifts of the FP fringes at certain magnetic fields [14,31,32], unlike $\Phi_{\mathrm{WKB}}$ and $\Phi_{\mathrm{AB}}$ yielding a continuous parabolic dispersion of the fringes with respect to the magnetic field [15]. Thus, the phasesensitive FP interference is a convenient tool to probe the Berry phase generated in the cavity.

We observe the FP interference under low magnetic fields $(|B| \leq 0.9 \mathrm{~T})$ by tuning $V_{\mathrm{tg}}$ at fixed $V_{\mathrm{bg}}=20 \mathrm{~V}$; see Fig. 3(a). Here, the oscillatory part of the conductance $G_{\text {osc }}$ is presented instead of the total conductance $G$ in order to circumvent the nonuniform conductance profile induced by the increasing magnetic field. We subtract a smoothed background $G_{0}\left(V_{\mathrm{tg}}\right)$ at each $B$ value and obtain the oscillatory part via $G_{\text {osc }}\left(V_{\mathrm{tg}}\right)=G\left(V_{\mathrm{tg}}\right)-G_{0}\left(V_{\mathrm{tg}}\right)$. The low-field dispersion of the FP fringes shows two distinct features. For $V_{\mathrm{tg}}$ close to the CNP $\left(V_{\mathrm{tg}}>-1.9 \mathrm{~V}\right)$, the FP fringes shift suddenly from their initial positions (e.g., yellow lines) to positions at slightly lower $V_{\text {tg }}$ (e.g., green lines) at $\left|B^{*}\right| \approx 0.15-0.25 \mathrm{~T}$, indicating that the Berry phase
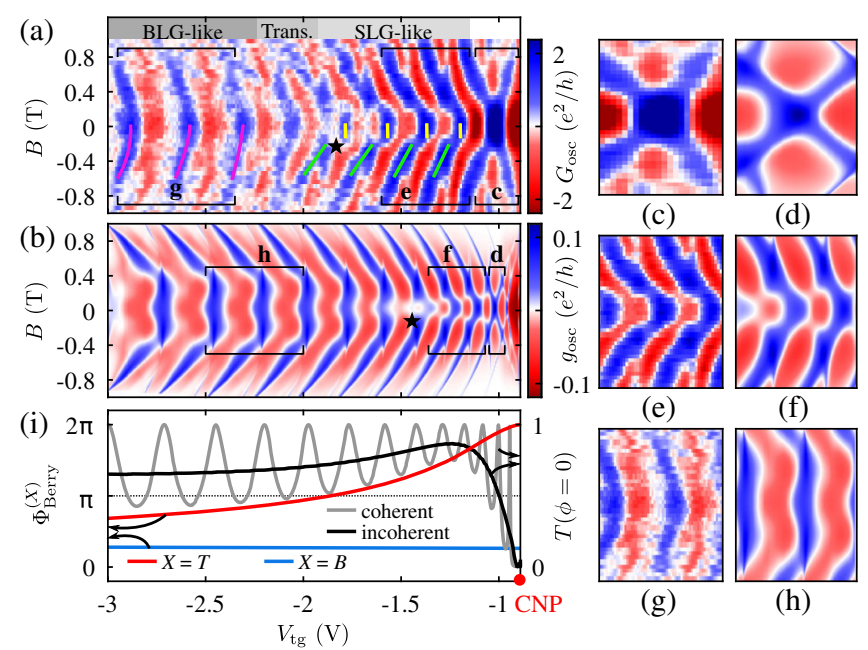

FIG. 3. (a) Fabry-Pérot interference measurements and (b) simulations at $V_{\mathrm{bg}}=20 \mathrm{~V}$ under low magnetic fields for device PNJ-B (conductance measurements at zero magnetic field are shown in the Supplemental Material [37]). The regions labeled by $\mathbf{c}$ to $\mathbf{h}$ in (a) and (b) are highlighted in the corresponding panels (c)-(h). (i) Berry phases for regions $B$ and $T$ are shown as blue and red curves, respectively. The corresponding transmission probability at normal incidence is calculated with phase-coherent (grey curve) and phase-incoherent (black curve) methods.

has been abruptly added to $\Delta \Phi[31,32,50,51]$. The amount of phase shift for each fringe increases with decreasing $V_{\text {tg }}$ and reaches $\pi$ at $V_{\mathrm{tg}} \approx-1.8 \mathrm{~V}$ [see the black star in Fig. 3(a)], suggesting that the Berry phase is continuously tuned across $\pi$ by modulating $V_{\mathrm{tg}}$. Here, the emergence of the Berry phase at $B^{*}$ instead of $B=0$ resembles the behavior of single-layer graphene (SLG), where the required geometric paths to acquire the Berry phase are formed with the assistance of low magnetic fields [31,32,50,51]. For $V_{\text {tg }}$ far away from the CNP $\left(V_{\mathrm{tg}}<-2.23 \mathrm{~V}\right)$, the FP fringes exhibit parabolic dispersion (marked by magenta lines) with respect to $B$, as expected for BLG [14]. This BLG-like dispersion without phase shift at $B^{*}$ illustrates that the Berry phase has already been included in $\Delta \Phi$ at $B=0$, highlighted by a transition region [labeled by "Trans." in Fig. 3(a)] between the BLG-like and SLG-like dispersions. The reason is that the trajectory of the wave vector $(k)$ forms a closed loop encircling the origin of momentum space, thus resulting in the nonzero Berry phase [15].

We have successfully reproduced the two types of dispersion, i.e., the SLG-like and the BLG-like, using quantum transport simulations based on a realistic electrostatic model, which is constructed from our experimental parameters but with a scattering region (the length $L=300 \mathrm{~nm}$ ) around the top gate; see Fig. 3(b). Note that $g_{\text {osc }}$ is the oscillatory part of the calculated single-mode conductance $g$ [35-37], and it is obtained using the same procedure as $G_{\text {osc }}$. For better comparison, the fringes in the 
regions labeled by $\mathbf{c}$ to $\mathbf{h}$ are highlighted in Figs. 3(c)-3(h). We found that the simulation result shows remarkable agreement with the experiment on the SLG-like [Figs. 3(e) and 3(f)] and BLG-like [Figs. 3(g) and 3(h)] dispersions under low magnetic fields, although the simulated patterns in Figs. 3(d), 3(f), and 3(h) occupy smaller regions in Fig. 3(b). In addition, the Berry phase of $\pi$ appears at $V_{\mathrm{tg}} \approx$ $-1.45 \mathrm{~V}$ (black star) in Fig. 3(b) instead of near $-1.8 \mathrm{~V}$ due to the reasonable differences between the realistic electrostatic model and the intricate experiments.

We calculate the Berry phase by a circular integral [14,15,21] for the gate range in Fig. 3(a) [see Fig. 3(i)]. The Berry phase in region $T\left(\Phi_{\text {Berry }}^{(T)}\right)$ is modulated from $2 \pi$ to $0.68 \pi$ while lowering $V_{\mathrm{tg}}$, which well accounts for the phase shifts in Fig. 3(a). Particularly, the Berry phase in region $T$ crosses $\pi$ at $V_{\mathrm{tg}}=-1.86 \mathrm{~V}$, which is consistent with the $\pi$-shift position in Fig. 3(a). Besides, the Berry phase in region $B\left(\Phi_{\text {Berry }}^{(B)}\right)$ is only affected by $V_{\mathrm{bg}}$ and takes a constant value of $0.28 \pi$ for $V_{\mathrm{bg}}=20 \mathrm{~V}$.

The quasiparticle tunneling in gapped BLG is simultaneously tuned as the Berry phase changes in region $T$. Given the variation of $\Phi_{\text {Berry }}^{(T)}$, we expect a transition from anti-Klein tunneling, corresponding to the Berry phase of $2 \pi$, to Klein tunneling at the Berry phase $\pi$, to reentrant anti-Klein tunneling upon further decreasing the Berry phase [15]. To demonstrate the anticipated transitions, the transmission probability at normal incidence $T(\phi=0)$ [see Fig. 3(i)] is investigated by quantum transport simulations for two cases: phase-coherent (grey curve) and phase-incoherent (black curve). The phase-coherent transmission probability oscillates due to the resonance condition. Instead, the phase-incoherent transmission probability suppresses the resonance and is calculated by the relation $T(\phi=0)=$ $1 /\left(1 / T_{L}+1 / T_{R}-1\right)$ [52], where $T_{L}$ and $T_{R}$ represent the transmission probability through the left and right $p n$ interfaces of the potential barrier, respectively. The resulting phase-incoherent $T(\phi=0)$ agrees with our expectation aside from two differences: (i) The Berry phase for anti-Klein tunneling, $\Phi_{\text {Berry }}^{(T)}=2 \pi$, appears at the CNP, where zero charge-carrier density in region $T$ also gives rise to the inhibition of transmission as anti-Klein tunneling. (ii) The maximum $T(\phi=0)$ reaches 0.87 at $V_{\mathrm{tg}}=-1.24 \mathrm{~V}$, which is close to the unity transmission probability for perfect Klein tunneling $[16,17,31,32]$. The factor that impedes the maximum $T(\phi=0)$ from reaching 1 is the Berry phase in region $B$, which is far from $\pi$. But perfect Klein tunneling requires the Berry phase to be $\pi$ in both the $T$ and $B$ regions. In addition, the reduction of $T(\phi=0)$ for $V_{\mathrm{tg}}<-1.24 \mathrm{~V}$ suggests that anti-Klein tunneling is partially restored. Therefore, the quasiparticle tunneling undergoes two processes: reaching Klein tunneling and recovering anti-Klein tunneling.

The transition from anti-Klein to Klein tunneling actually relies on the modulation of pseudospin orientation in gapped BLG. When the Fermi level is tuned close to the band edge, the pseudospin is rotated out of plane [see Fig. 1(b)], leading to broken chirality $[15,19,20,22]$. The momentum of charge carriers is, therefore, unlocked to the pseudospin, allowing Klein tunneling in gapped BLG [see Fig. 1(f)]. Even though the chirality sustains Klein tunneling in SLG [16,17], the contrary happens in gapped BLG; i.e., Klein tunneling favors the impaired chirality. On the other hand, the chirality can be restored in gapped BLG [15], as long as the pseudospin recovers its in-plane orientation at sufficiently high Fermi energies; at the same time, the Berry phase of $2 \pi$ (or equivalently 0 ), as well as anti-Klein tunneling, is regained. The recovery of antiKlein tunneling is affected by two parameters-namely, the interlayer asymmetry and the Fermi energy. The chirality is broken because of the increasing interlayer asymmetry but recovered due to the rising Fermi energy.

SLG-like and BLG-like Berry phase.-Figures 4(a) and 4(b) show the FP interference patterns magnified from the white rectangles in Figs. 2(c) and 2(d), respectively. Both experiments and simulation in the $n \bar{p} n n$ regime show nearly half-period shifts of the FP fringes, for example, highlighted by the green dashed lines in Figs. 4(a) and 4(b). The nearly half-period shifts indicate that a phase change of about $\pi$ is suddenly incorporated in the phase difference $\Delta \Phi$. This phase shifts arise from two different ways, i.e., the SLG-like and the BLG-like, to acquire the Berry phase in gapped BLG. For better interpretation, we calculate the Berry phase in region $T$ as a function of $V_{\mathrm{tg}}$ and $V_{\mathrm{bg}}$ [14] [see Fig. 4(c)]. The phase-shift positions in Fig. 4(a) are labeled as three green dots near the $\pi$ line on Fig. 4(c), which arrange along the dotted line. The BLG-like $\Phi_{\text {Berry }}^{(T)}$ can be acquired at zero magnetic field; hence, it appears as
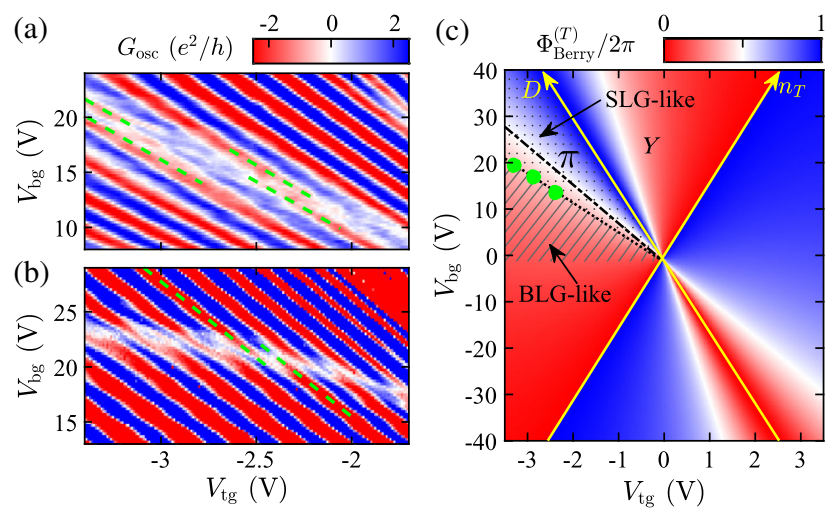

FIG. 4. Fabry-Pérot interferences at zero magnetic field are shown both (a) experimentally and (b) theoretically, zoomed in on the white rectangle of Figs. 2(c) and 2(d), respectively. (c) Calculation of the Berry phase in region $T$ as a function of $V_{\mathrm{tg}}$ and $V_{\mathrm{bg}}$. The dash-dotted line shows the position of $\Phi_{\text {Berry }}^{(T)}=\pi$. The green dots mark the phase-shift positions that appear in (a) and define the dotted line. The displacement field axis $(D)$ and the charge-carrier density axis in region $T\left(n_{T}\right)$ are shown in yellow. 
the predicted values in the stripe-shaded region of Fig. 4(c). However, the SLG-like $\Phi_{\text {Berry }}^{(T)}$ needs low magnetic fields to develop and is unavailable at $B=0$. Actually, $\Phi_{\text {Berry }}^{(T)}$ in the dot-shaded region remains zero instead of the calculated value. Accordingly, the phase shifts of about $\pi$ show up around the intersection between the SLG-like and BLGlike regions, i.e., the dotted line, and directly prove the existence of two mechanisms, SLG-like and BLG-like, to obtain the Berry phase in gapped BLG. Additionally, although $\Phi_{\text {Berry }}^{(T)}$ in the $Y$ region between the two yellow axes in Fig. 4(c) varies similarly as in the $n \bar{p} n n$ regime, the nearly half-period phase shifts do not appear due to the absence of FP interferences in the $n \bar{n} n n$ regime.

Conclusion.-We have examined the quasiparticle tunneling as well as the related Berry phase in BLG using a Fabry-Pérot interferometer based on a dual-gated geometry. As the crystal inversion symmetry is broken by applying a displacement field, full control of the Berry phase within the range $0.68 \pi-2 \pi$ is achieved by manipulating the Fermi energy of charge carriers. Two distinct ways to acquire the Berry phase, SLG-like and BLG-like, coexist and can be switched for each other. Consequently, the corresponding quasiparticle tunneling undergoes a transition from antiKlein to almost complete Klein tunneling with a maximum transmission probability of 0.87 at normal incidence. Therefore, in gapped BLG, tuning from BLG-like antiKlein tunneling to SLG-like Klein tunneling is reachable by appropriate electrical gating.

We acknowledge A. Varlet for fruitful discussions. Financial supports from the Deutsche Forschungsgemeinschaft (DFG) within SFB No. 689, Project No. DA 1280/3-1, Project No. Ri 681-13/1, and the program of the Forschungsgroßgeräte No. 121384/17-1; from the Taiwan Minister of Science and Technology (MOST) under Grant No. 107-2112-M-006-004-MY3; from the Helmholtz association through the program STN; and from the DFG Center for Functional Nanostructures (CFN) are gratefully acknowledged.

\footnotetext{
*Renjun.Du@outlook.com

†minghao.liu@phys.ncku.edu.tw

"romain.danneau@kit.edu
}

[1] K. S. Novoselov, A. K. Geim, S. V. Morozov, D. Jiang, M. I. Katsnelson, I. V. Grigorieva, S. V. Dubonos, and A. A. Firsov, Nature (London) 438, 197 (2005).

[2] A. K. Geim and K. S. Novoselov, Nat. Mater. 6, 183 (2007).

[3] A. H. C. Neto, F. Guinea, N. M. R. Peres, K. S. Novoselov, and A. K. Geim, Rev. Mod. Phys. 81, 109 (2009).

[4] Y. Liu, G. Bian, T. Miller, and T.-C. Chiang, Phys. Rev. Lett. 107, 166803 (2011).

[5] S. V. Morozov, K. S. Novoselov, M. I. Katsnelson, F. Schedin, D. C. Elias, J. A. Jaszczak, and A. K. Geim, Phys. Rev. Lett. 100, 016602 (2008).
[6] K. S. Novoselov, E. McCann, S. V. Morozov, V. I. Fal'ko, M. I. Katsnelson, U. Zeitler, D. Jiang, F. Schedin, and A. K. Geim, Nat. Phys. 2, 177 (2006).

[7] E. McCann, Phys. Rev. B 74, 161403 (2006).

[8] E. McCann and M. Koshino, Rep. Prog. Phys. 76, 056503 (2013).

[9] J. B. Oostinga, H. B. Heersche, X. Liu, A. F. Morpurgo, and L. M. K. Vandersypen, Nat. Mater. 7, 151 (2008).

[10] Y. Zhang, T.-T. Tang, C. Girit, Z. Hao, M. C. Martin, A. Zettl, M. F. Crommie, Y. R. Shen, and F. Wang, Nature (London) 459, 820 (2009).

[11] T. Taychatanapat and P. Jarillo-Herrero, Phys. Rev. Lett. 105, 166601 (2010).

[12] M. V. Berry, Proc. R. Soc. Lond. A 392, 45 (1984).

[13] Y. Zhang, Y.-W. Tan, H. L. Stormer, and P. Kim, Nature (London) 438, 201 (2005).

[14] A. Varlet, M.-H. Liu, V. Krueckl, D. Bischoff, P. Simonet, K. Watanabe, T. Taniguchi, K. Richter, K. Ensslin, and T. Ihn, Phys. Rev. Lett. 113, 116601 (2014).

[15] A. Varlet, M.-H. Liu, D. Bischoff, P. Simonet, T. Taniguchi, K. Watanabe, K. Richter, T. Ihn, and K. Ensslin, Phys. Status Solidi RRL 10, 46 (2016).

[16] M. I. Katsnelson, K. S. Novoselov, and A. K. Geim, Nat. Phys. 2, 620 (2006).

[17] M. I. Katsnelson, Graphene: Carbon in Two Dimensions (Cambridge University Press, Cambridge, England, 2012).

[18] V. Kleptsyn, A. Okunev, I. Schurov, D. Zubov, and M. I. Katsnelson, Phys. Rev. B 92, 165407 (2015).

[19] H. Min, G. Borghi, M. Polini, and A. H. MacDonald, Phys. Rev. B 77, 041407 (2008).

[20] A. H. MacDonald, J. Jung, and F. Zhang, Phys. Scr. 2012, 014012 (2012).

[21] D. Xiao, M. C. Chang, and Q. Niu, Rev. Mod. Phys. 82, 1959 (2010).

[22] M. B. Lundeberg and J. A. Folk, Science 346, 422 (2014).

[23] F. Ghahari, D. Walkup, C. Gutiérrez, J. F. Rodriguez-Nieva, Y. Zhao, J. Wyrick, F. D. Natterer, W. G. Cullen, K. Watanabe, T. Taniguchi, L. S. Levitov, N. B. Zhitenev, and J. A. Stroscio, Science 356, 845 (2017).

[24] P. San-Jose, E. Prada, E. McCann, and H. Schomerus, Phys. Rev. Lett. 102, 247204 (2009).

[25] D. Xiao, W. Yao, and Q. Niu, Phys. Rev. Lett. 99, 236809 (2007).

[26] W. Yao, D. Xiao, and Q. Niu, Phys. Rev. B 77, 235406 (2008).

[27] R. V. Gorbachev, J. C. W. Song, G. L. Yu, A. V. Kretinin, F. Withers, Y. Cao, A. Mishchenko, I. V. Grigorieva, K. S. Novoselov, L. S. Levitov, and A. K. Geim, Science 346, 448 (2014).

[28] Y. Shimazaki, M. Yamamoto, I. V. Borzenets, K. Watanabe, T. Taniguchi, and S. Tarucha, Nat. Phys. 11, 1032 (2015).

[29] N. Nagaosa, J. Sinova, S. Onoda, A. H. MacDonald, and N. P. Ong, Rev. Mod. Phys. 82, 1539 (2010).

[30] Z. Qiao, W. Ren, H. Chen, L. Bellaiche, Z. Zhang, A. H. MacDonald, and Q. Niu, Phys. Rev. Lett. 112, 116404 (2014).

[31] A. V. Shytov, M. S. Rudner, and L. S. Levitov, Phys. Rev. Lett. 101, 156804 (2008).

[32] A. F. Young and P. Kim, Nat. Phys. 5, 222 (2009).

[33] L. E. F. F. Torres, S. Roche, and J. C. Charlier, Introduction to Graphene-Based Nanomaterials: From Electronic 
Structure to Quantum Transport (Cambridge University Press, Cambridge, England, 2014).

[34] L. Wang, I. Meric, P. Y. Huang, Q. Gao, Y. Gao, H. Tran, T. Taniguchi, K. Watanabe, L. M. Campos, D. A. Muller, J. Guo, P. Kim, J. Hone, K. L. Shepard, and C. R. Dean, Science 342, 614 (2013).

[35] P. Rickhaus, R. Maurand, M.-H. Liu, M. Weiss, K. Richter, and C. Schönenberger, Nat. Commun. 4, 2342 (2013).

[36] M.-H. Liu, J. Bundesmann, and K. Richter, Phys. Rev. B 85, 085406 (2012).

[37] See the Supplemental Material at http://link.aps.org/ supplemental/10.1103/PhysRevLett.121.127706 for a full description of the sample information, electrostatic model, Fabry-Pérot interferences, the Berry phase and quasiparticle tunneling, which includes Refs. [38-46].

[38] V.E. Calado, S. Goswami, G. Nanda, M. Diez, A. R. Akhmerov, K. Watanabe, T. Taniguchi, T. M. Klapwijk, and L. M. K. Vandersypen, Nat. Nanotechnol. 10, 761 (2015).

[39] M. Ben Shalom, M. J. Zhu, V. I. Fal'ko, A. Mishchenko, A. V. Kretinin, K. S. Novoselov, C. R. Woods, K. Watanabe, T. Taniguchi, A. K. Geim, and J. R. Prance, Nat. Phys. 12, 318 (2016).

[40] D. K. Cheng, Field and Wave Electromagnetics, 2nd ed. (Prentice Hall, Englewood Cliffs, NJ, 1989).
[41] M.-H. Liu, Phys. Rev. B 87, 125427 (2013).

[42] M.-H. Liu, J. Comput. Electron. 12, 188 (2013).

[43] P. A. Khomyakov, G. Giovannetti, P. C. Rusu, G. Brocks, J. van den Brink, and P. J. Kelly, Phys. Rev. B 79, 195425 (2009).

[44] P. A. Khomyakov, A. A. Starikov, G. Brocks, and P. J. Kelly, Phys. Rev. B 82, 115437 (2010).

[45] J. W. McClure, Phys. Rev. 108, 612 (1957).

[46] J. C. Slonczewski and P. R. Weiss, Phys. Rev. 109, 272 (1958).

[47] A. Logg, K.-A. Mardal, and G. N. Wells, Automated Solution of Differential Equations by the Finite Element Method (Springer, New York, 2012).

[48] C. Geuzaine and J.-F. Remacle, Int. J. Numer. Methods Eng. 79, 1309 (2009).

[49] M.-H. Liu, P. Rickhaus, P. Makk, E. Tóvári, R. Maurand, F. Tkatschenko, M. Weiss, C. Schönenberger, and K. Richter, Phys. Rev. Lett. 114, 036601 (2015).

[50] M. Ramezani Masir, P. Vasilopoulos, and F. M. Peeters, Phys. Rev. B 82, 115417 (2010).

[51] M.-H. Liu and K. Richter, Phys. Rev. B 86, 115455 (2012).

[52] S. Datta, Electronic Transport in Mesoscopic Systems (Cambridge University Press, Cambridge, England, 1997). 